\title{
Surface Growth Modes analysed with Modern Microscopic and Computing Techniques
}

\author{
Prof. Dr. rer. nat. Asta Richter, Prof. Dr. Roger Smith
}

\begin{abstract}
It is demonstrated how new microscopes with atomic resolution in combination with modern fast computers and computational techniques can be used in a complementary way in the analysis and explanation of crystal growth on surfaces. Examples are given of spiral formation, fractal growth, fullerene formation and the growth of $\mathrm{C}_{60}$ films.
\end{abstract}

Es wird gezeigt, wie neue Mikroskopiertechniken mit atomarer Auflösung in Kombination mit modernen schnellen Computern und neuen Computertechniken genutzt werden, um Kristallwachstum auf Oberflächen zu analysieren und zu erklären. Beispiele für Spiralformationen, Fraktalwachstum, Fullerenebildung und Wachstum von $\mathrm{C}_{60}$ Filmen werden diskutiert.

\section{Introduction}

The way in which crystals grow has long been a subject of fascination to scientists but the advancement of computational power together with new microscopes with atomic resolution has enabled the study of the nucleation of the growth processes in a way which was not previously possible. We intend to demonstrate in this paper how microscopy together with computational physics can be used to observe and explain a number of atomic-scale dynamical processes at crystal surfaces. Computer simulations enable us to calculate dynamical processes which can be used together with the images obtained in the microsopes with atomic resolution in an understanding of fundamental phenomena at crystal surfaces.

The Scanning Tunnelling Microscope (STM) was invented by Binnig et al [1]. The principle of operation is relatively simple. A sharp (tungsten) tip is placed next to a conducting surface, so close that the electronic wave functions of atoms at the tip overlap with those in the surface. A potential difference is applied beween the surface and the tip and a tunneling current flows. By varying the potential and/or the separation, the local density of states may be probed. By scanning the tip over a surface, a picture of the topography can be formed by variations in the tunnelling current or from variations in tip height necessary to maintain constant tunnelling current and therefore constant tip/surface separation.

The STM therefore will only work with conducting surfaces. Atomic Force Microscope (AFM) emerged from
STM technology and can work with non-conductors. The principle of operation depends on the minute atomic interaction force beween the atoms at the end of the tip and the surface. The tip is held on a sensitive cantilever whose deflection is magnified by an optical lever to measure this force. The same scanning technique is used as for the STM and the AFM can also achieve atomic resolution. The AFM images produced in this paper are those taken with a Nanoscope III instrument. This has exchangeable measuring heads for STM and AFM. Microscopes such as the Nanoscope instrument can be used with great success to look at the atomic structure and topography of solid surfaces and as such are extremely useful tools in surface physics.

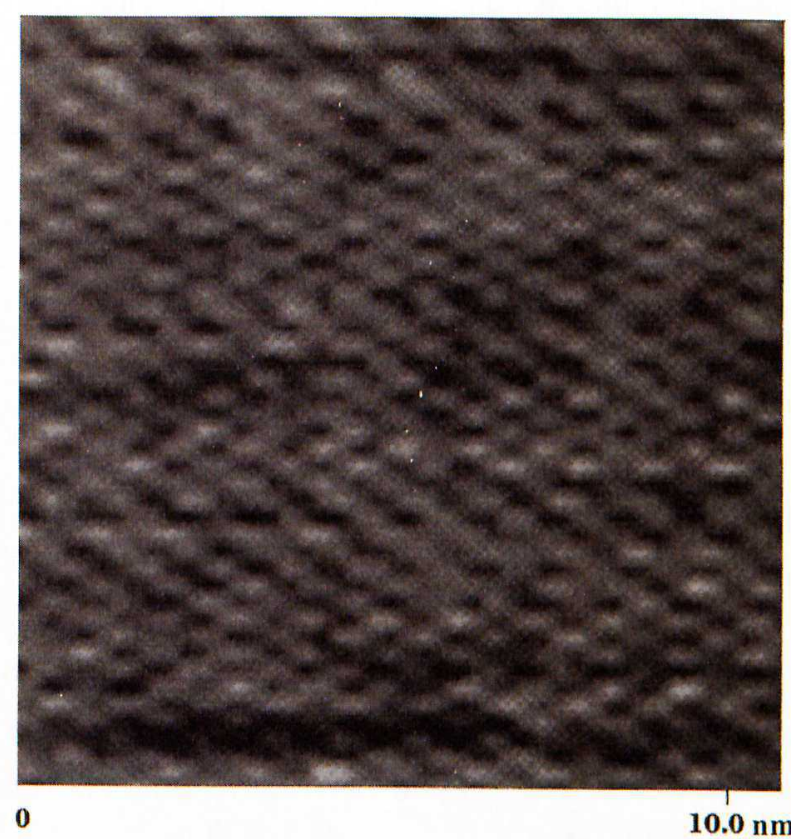

Figure 1: An AFM image of a $\mathrm{NaCl}$ crystal surface showing atomic resolution.

Figure 1. shows a $\{100\} \mathrm{NaCl}$ crystal surface imaged with this instrument (in the AFM mode because $\mathrm{NaCl}$ is non-conducting). the micrograph shows the cubic symmetry of the crystal structure and from the atomic corrugations the lattice constant is roughly estimated to be $5.4 \AA$ compared to a reported value in the literature of $5.6 \AA$. The corrugations observed are due to the $\mathrm{Cl}$ ions alone. The $\mathrm{Na}$ atoms cannot be observed due to stick-slip processes [2].

It is often important to know the structure and topography of surfaces. A surface is where many important processes happen, from Catalysis and Adhesion through to Lubrication and Erosion. If a perfect crystal is cleaved, the surface often does not remain in the structure of the 
bulk-terminated solid. For example, the most stable form of the $\{111\}$ surface of silicon is a $7 \times 7$ reconstruction. The $\{100\}$ surface undergoes a $2 \times 1$ dimer reconstruction. If the surface is cleaved in air rather than a vacuum, the dangling bonds can absorb hydrogen or oxygen rather than reconstruct. These are all examples of atomic scale phenomena of the order of Angstroms in dimension but small scale atomic processes can often combine together to produce strange topographies on surfaces of the order of nanometers or microns. This might happen, for example, in thin film growth or surface erosion by interaction with energetic ions. The type of topography produced by these processes is usally impossible to predict in advance and it is often quite exciting to see for the first time a new image in one of these microscopes. The results can be totally unexpected.

The advances in computational power add another tool to scientific investigation in addition to the classical methods of experimentation and analytical theory: -- the tool of computer simulation. A simulation is neither a theory nor an experiment but contains elements of both. Although most scientists would claim to understand the term „computer simulation" there is no totally satisfactory definition. A simulation is not a theory but a numerical model of a system. If it is a good model one may explore the behaviour of the real system by changing the numerical value of its input parameters and noting the changed responses.

Surface physics readily lends itself to investigation by computer simulation and in this paper we will give examples of unusual topographies which we have observed in the STM/AFM and use three different computational techniques, Cellular Automata, Monte-Carlo and Molecular Dynamics, to explain these phenomena. We will describe in general terms the computational models that can be used to explain the specific experiments we have chosen. The main point here will be to demonstrate that the two techniques combined together can explain much of the basic science behind many observed phenomena.

\section{Spiral Formation at Defects}

Figure 2 gives an example of an AFM image which shows where a spiral structure has formed on the surface of a $\mathrm{NaCl}$ crystal. Spirals can either grow as structures protruding from the surface as a result of atoms being added to a crystal or they can form as small pits on a surface due to heating and removal of atoms. Atoms can be preferentially removed from a surface at defects or edges by heating in the opposite way to which growth occurs. The micrograph shown in Figure 2 has formed as a result of evaporation of atoms from a surface (i.e. the structure is a pit). In this case the arms of the spiral are not ledges of single atoms but terraces which are a few atomic layers ( $<10$ layers) in depth and the bottom of the pit has a radius of several hundred atoms.

The ledges are larger than one atomic layer either because of diffusion or the extended nature of the defect. The bottom of the pit is flat indicating that the defect

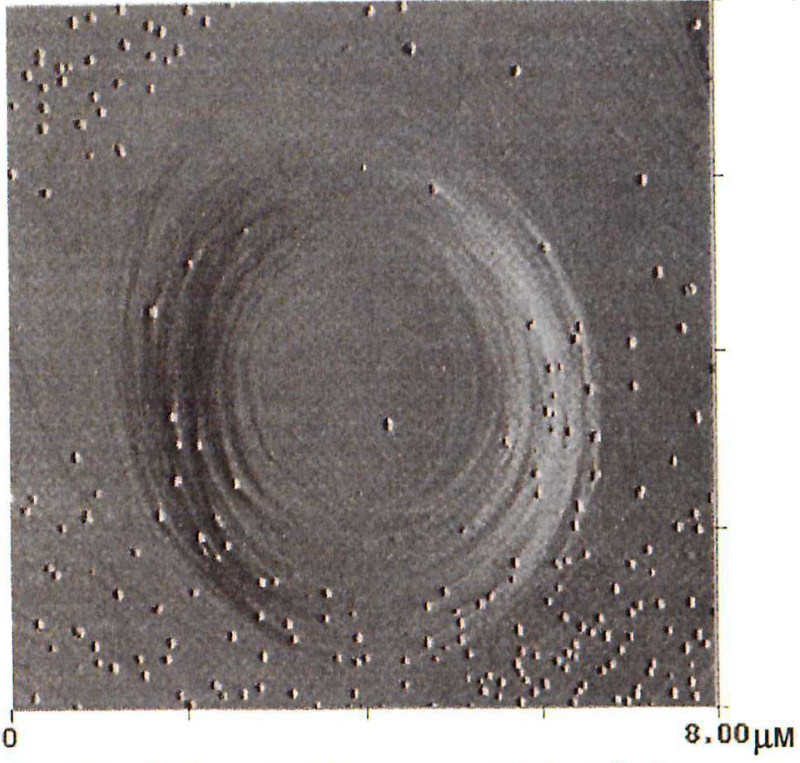

Figure 2: An AFM image of spiral formation on a NaCl crystal surface.

is no longer present to act as a source to remove deeper layers. Using the depth information in the AFM, the depths of the pits and hence a rough estimate of the length scale of the original defects in the crystal can be estimated.

Crystal growth occurs when particles arrive at a surface and migrate until they arrive at a position from which they no longer move. The probability that a particle may stick at a site depends on the local property of the site. For example an edge is a likely stable site because each atom there has fewer neighbours than in the bulk. A site which has two adjacent edges is likely to have an even higher probability of capturing a diffusing particle. It was these kinds of ideas that led Frank [3] to propose his explanation for the spiral growth which can occur at screw dislocations. In this explanation the particles are individual atoms and these only stick to the edges within a plane. A new plane is initiated only where the screw dislocation intersects the surface. Frank's model can be formulated to give a very nice example of what computer scientists now call a Cellular Automaton. Cellular automata are discrete (dynamical) systems whose behaviour is completely specified in terms of a local relation. They are the computer scientist's counterpart to the physicist's concept of field [4]. Over recent years, specialist computers called Cellular Automata Machines have been built to compute efficiently models built using the discrete particle approach. Such machines consist of large numbers of parallel processors which exploit the simplicity and the local nature of the model. For large field problems the speed gained by this parallelism is essential but for the simple problem described here it is not essential. In a true cellular automaton therefore, all the cells are updated simultaneously. If the cells are updated sequentially with a predefined statistical probability then this would be usually described as a Monte-Carlo simulation.

Spiral growth at a screw dislocation gives an example of a true cellular automaton. The various stages in the simulations are shown in Figure 3a for modelling such spiral growth. The algorithm is extremely simple. The 
bulk material is described by an array of cubic cells. At each step a new cell is added only at (a) those positions which have an adjacent filled vertical face or (b) at the edge of the dislocation on the next surface provided all adjacent cells at the lower level are filled. The second of these mechanisms gives rise to growth on the next layer. The first six steps of this process are shown in
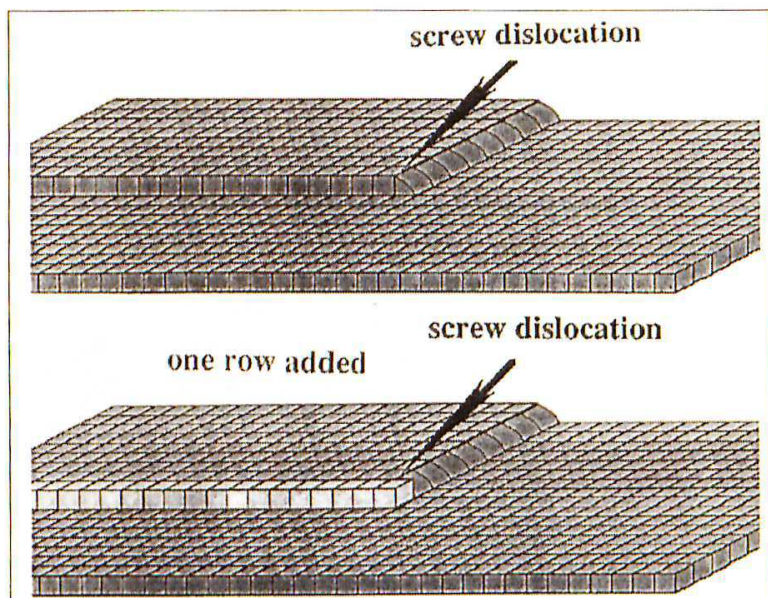

screw dislocation

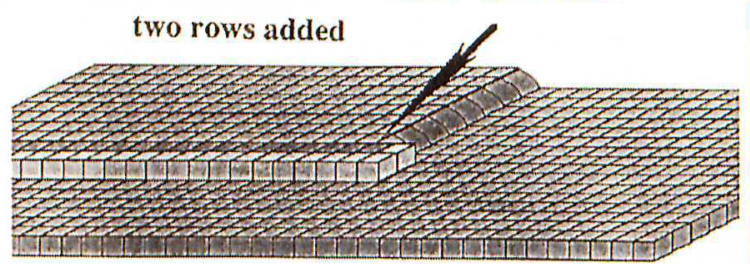

screw dislocation
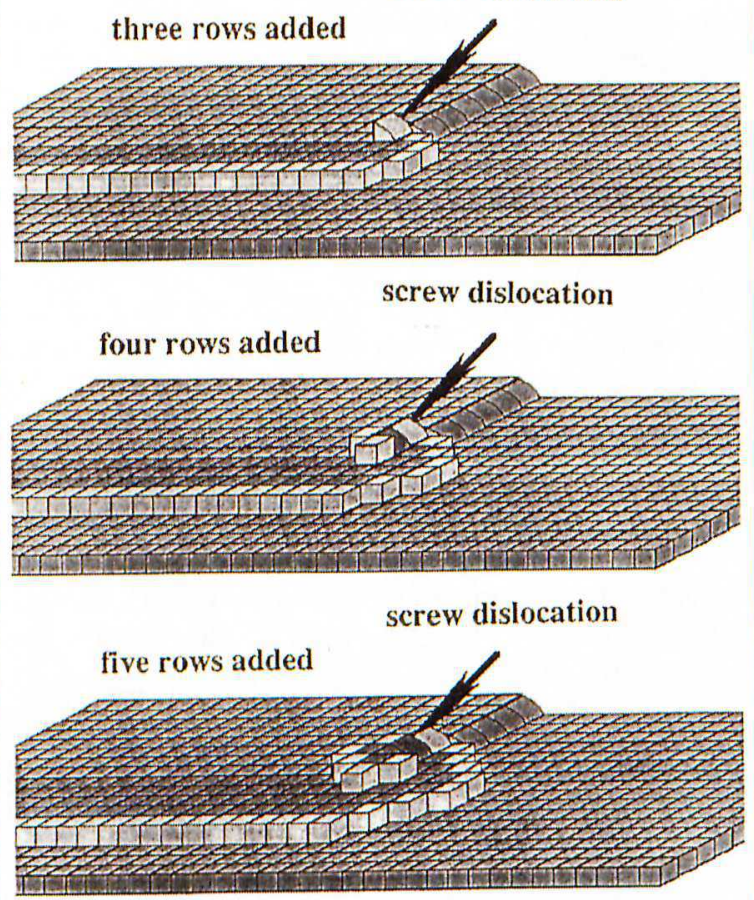

screw dislocation

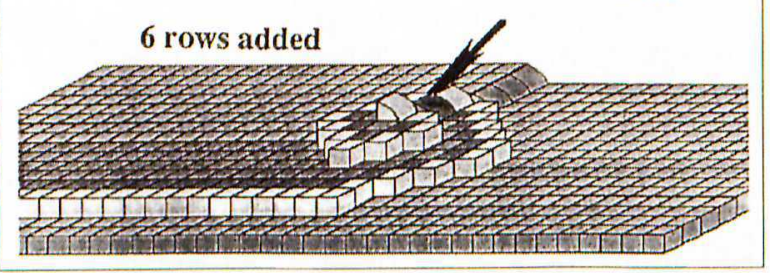

Figure 3a: A cellular automaton model of spiral growth at screw dislocation. figure 3a. Figure 3b shows the development of a spiral pit after evaporation of six layers using the same model as described in Figure 3a to remove atoms from a surface rather than add them. This figure bears a close qualitative resemblance to the experimental results shown in Figure 2.

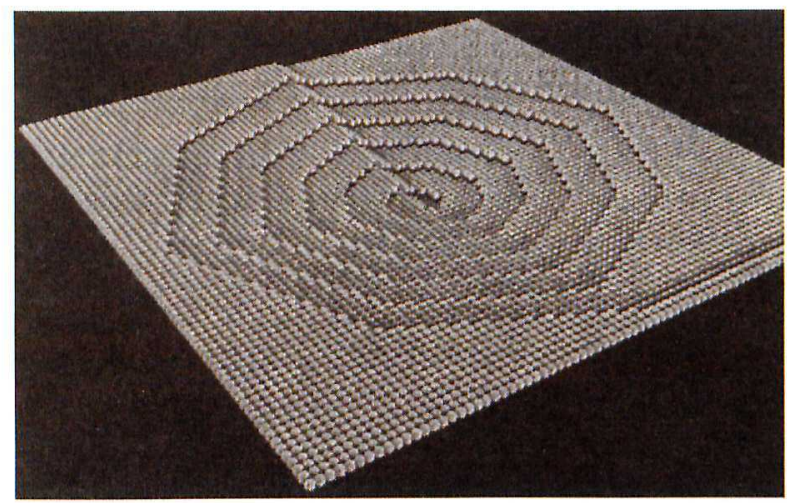

Figure 3b: The same model, except now used for evaporation after the removal of 6 layers.

\section{Growth of Au islands on metal surfaces}

Observations in the STM have shown that the initial grwoth in the sub monolayer range of Au on clean metal crystal surfaces can occur in the form of fractal islands $[5,6]$. Gold atoms are highly mobile over the surfaces but unlike the growth process described above in section (1) in the sub-monolayer range, the growth at the seed points is two-dimensional. This usually occurs at room temperature when the coverage of the substrate is low. At higher temperatures the fractal islands anneal and the dendritic arms disappear. An STM of Au grown on $\operatorname{Ru}\{0001\}$ is shown in Figure 4. The fractal dimension of the structures seems to vary according to the experimental conditions such as the arrival rate of the Au atoms and the temperature of the substrate. Hwang et al. [5] report a fractal dimension of $1.72 \pm 0.07$ for Au grown on Ru\{0001\}. We have also carried out experiments of $\mathrm{Au}$ growth on both $\mathrm{Si}$ and $\mathrm{NaCl}$. In both cases similar fractal structures grow as on Ru. However, our own experiments indicate that there is a wider range of fractal dimension which does not seem to have a simple relation to the growth conditions [6].

The model of the growth process is that of diffusion limited aggregation (DLA) [7]. The Au atoms become trapped in the surface binding potential but are able to move two-dimensionally across the surface until they enter the vicinity of a fixed Au atom where they bind. This can be easily described by the following Monte-Carlo computer algorithm.

(1) First set up a two dimensional cellular structure. the simplest are usually square or hexagonal. In the simulations shown in Figure 5 the network is hexagonal.

(2) Take a circle a large fixed distance from the fractal structure, centred at the centre of mass of the fractal island.

(3) Take a random position on this circle and let a particle undertake a random walk from the circle. If it strays outside a circle of a pre-defined larger radius then start 
again. Otherwise continue until it reaches the fractal structure where it sticks.

(4) Repeat the process from (2).

A fractal structure grown in this way is shown in Figure 5 a which shows a remarkable similarity to the micrograph, Figure 4. Figure 5b shows the same structure except that arriving particles have a radius 3 times the cell diameter. Here the fine structure of the fractal tree is lost. Structures such as these have been observed at higher temperatures.

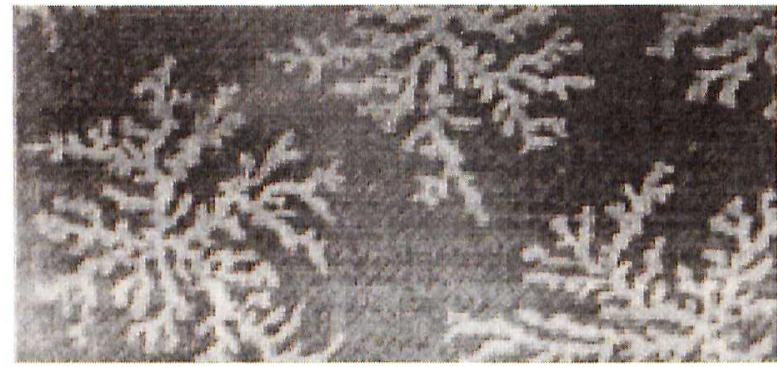

Figure 4: Initial stages of growth of a gold film on $R u\{0001\}$ at room temperature. The flux is approximately $0.2 \mathrm{ML}$. The radii of the fractal structures are between 300 and $400 \mathrm{~nm}$.
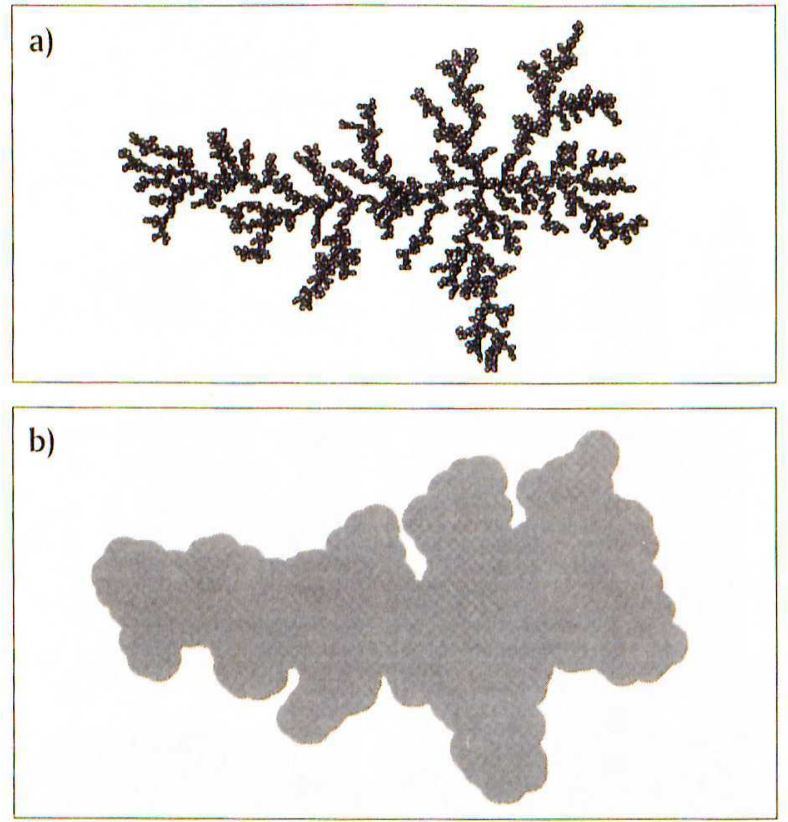

Figure 5: Two-dimensional DLA fractal growth. (a) To match the STM in Figure 4 more exactly, the particles are drawn slightly bigger than the size of the hexagonal cell. (b) Fractal growth with circular particles which have a radius of 3 times the diameter of the cell. In this case the individual details of the fractal trees become smeared.

\section{Fullerene Formation and the Growth of Fullerene Films}

An important scientific discovery of the 1980's was the new form of carbon called fullerene. This material has attracted interest by many groups and we have prepared molecular fullerene and in addition made thin films of this material. The $\mathrm{C}_{60}$ molecule itself is best prepared by passing an electrical current between two graphite electrodes in a helium or argon atmosphere. Optimum prodution is achieved when there is a background He pressure of about 100 torr. Before describing our experiments in producing thin $\mathrm{C}_{60}$ films it should also be mentioned that the formation of these molecules can also be studied by the third and potentially most powerful computational technique, Molecular Dynamics. The technique is in essence simple but its application is in practise much more complicated than the models described in sections 2 and 3 above. Newton's laws of motion are integrated numerically for systems involving large numbers of particles. What makes the simulations attractive and more realistic than before is the advent of many-body potentials and the increase in computing power that makes large simulations feasible.

Figure 6 shows a computer simulation of the condensation of $120 \mathrm{C}$ particles into a $\mathrm{C}_{120}$ molecule by interaction with 30 argon atoms in a confined volume. The formation process appears to be complex. First small chains form which eventually close into large rings. The rings can wrap around each other to form a three dimensional structure which eventually anneals into a $C_{120}$ cage. This simulation took many days to run on a fast UNIX workstation and even then is an idealised model of the fullerene formation process which in the real world takes place with a larger $\mathrm{Ar}$ to $\mathrm{C}$ atom ratio and atoms distributed with a much lower density. Nonetheless there are hints here that the fullerene formation process does not occur in a symmetrical way but that the molecules condense from unsymmetric three-dimensional structures formed by the wrapping round of large rings.

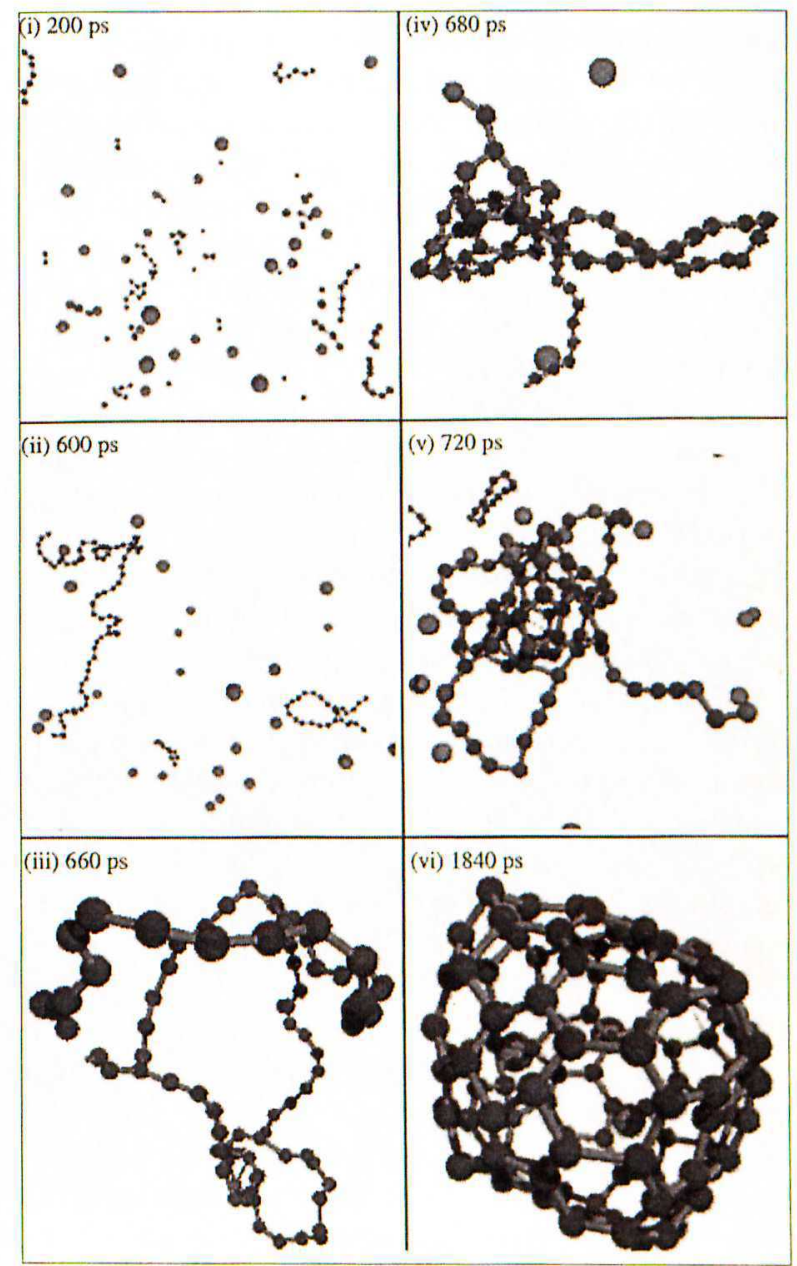

Figure 6: Snapshots during fullerene formation. The system consisted of 120 carbon particles and $30 \mathrm{Ar}$ atoms initially at $5000 \mathrm{~K}$. The Ar atoms were coupled to a $2000 \mathrm{~K}$ Berendsen [8] bath with a time constant of $1 \mathrm{ps}$. 
The electronic and structural properties of well ordered films of fullerenes is a subject of both scientific and potential technological importance and because of the potential applications of this new material, the deposition experiments with fullerenes have been carried out on many diverse materials. The films were grown in the laboratory by thermal evaporation of $\mathrm{C}_{60}$ on various substrates, under ultra high vacuum conditions. The $\mathrm{C}_{60}$, in powder form, was placed in a crucible and evaporated at a temperature of between 400 and $500^{\circ} \mathrm{C}$. The growth rate was approximately $0.01 \mathrm{~nm} / \mathrm{s}$ and the films were all grown at different thicknesses. Here we concentrate on examining the initial stages of the growth process on different substrates. The substrate temperature was varied from room temperature up to $250^{\circ} \mathrm{C}$ in order to study its effect on the grain size and surface roughness. STM and AFM pictures of the film growth on silicon, quartz and mica are shown in Figures 7, 8, and 9. In Figure 7 the initial stage of growth on Si $\{100\}$ (2x1) with a coverage of approximately one monolayer is shown. It is clearly seen that both the $(3 \times 4)$ and $(4 \times 4)$ packings are present [9]. Figure 8 shows growth on mica at different temperatures. At room temperature (a)-(c), the molecules do not diffuse large distances across the surface. Amorphous $\mathrm{C}_{60}$ films are formed without a crystal structure. Neither island growth at the beginning nor layer by layer growth occurs. With a certain thickness of the $C_{60}$ film self-formation of the $C_{60}$ grains starts. After the deposition of $7 \mathrm{~nm}$ (7 layers), grains of approximately $50 \mathrm{~nm}$ in diameter are visible. At substrate temperatures above $200^{\circ} \mathrm{C}$, island growth is immediately visible due to higher mobility of the molecules across the surface. After an average deposition equivalent to 2 molecular layers, islands of the order of $250 \mathrm{~nm}$ in diameter and up to 5 molecules in height are observed. As more material is added, the islands coalesce and the growth mode changes to a layer by layer mode, Figure 8e. By the time 7 molecular layers have been added, a uniform film with roughness at most one or two layers has occured, Figure $8 \mathrm{f}$. The grains become both larger in diameter and flatter with increasing substrate temperature. The structures which occur in Figure $8 \mathrm{f}$ bear a close resemblance to the simulation shown in Figure 5b for modified fractal growth.

For quartz, the film growth is initially amorphous at room temperature, Figure 9a, but after the deposition of 10 layers, Figure $9 \mathrm{~b}$, round island clusters are present about $50 \mathrm{~nm}$ in diameter. The films themselves have a roughness of $5 \mathrm{~nm}$. At $250^{\circ} \mathrm{C}$, larger islands appear and after the deposition of 10 layers, Figure $9 d$, these are double the lateral size of those at room temperature, Figure 9c. The large number of atoms required to produce topographic features on this scale means that modelling is currently beyond the limits of MD computer simulations. However the initial stages of growth can be examined.

In order to model the growth process it is necessary to have a good description of the inter atomic forces between the atoms. For MD modelling of dynamic processes in covalent materials e.g. Si or C many-body semiempirical potential functions such as those due to Tersoff [10] and Brenner [11] are often used. In a film

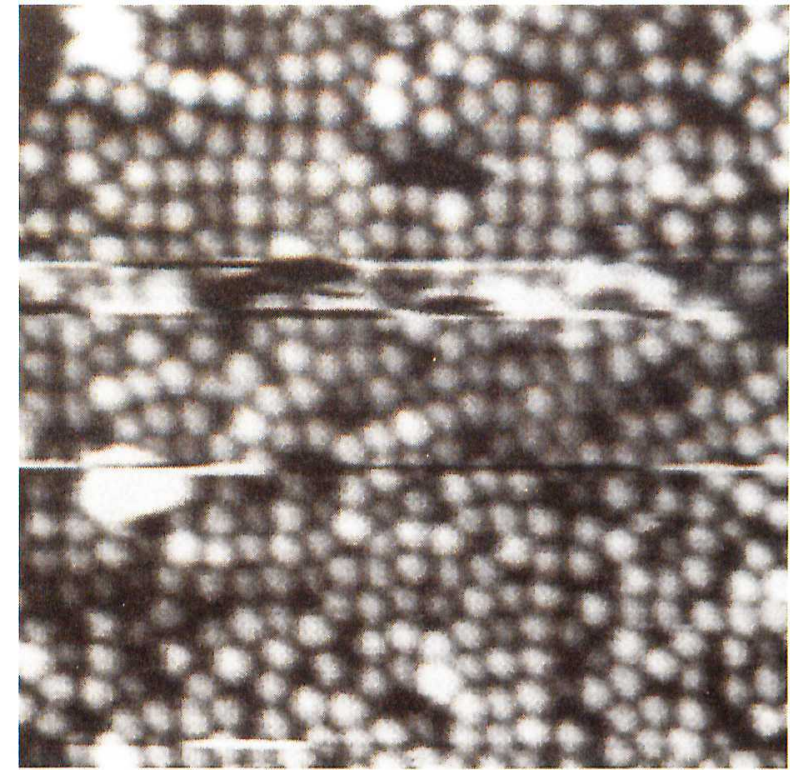

Figure 7: STM image of the Si $\{100\}(2 \times 1)$ surface with coverage of approximately 1 monolayer, showing both the $c(3 \times 4)$ and $c(4 \times 4)$ packings. The dark regions are bare regions of the Si surface.
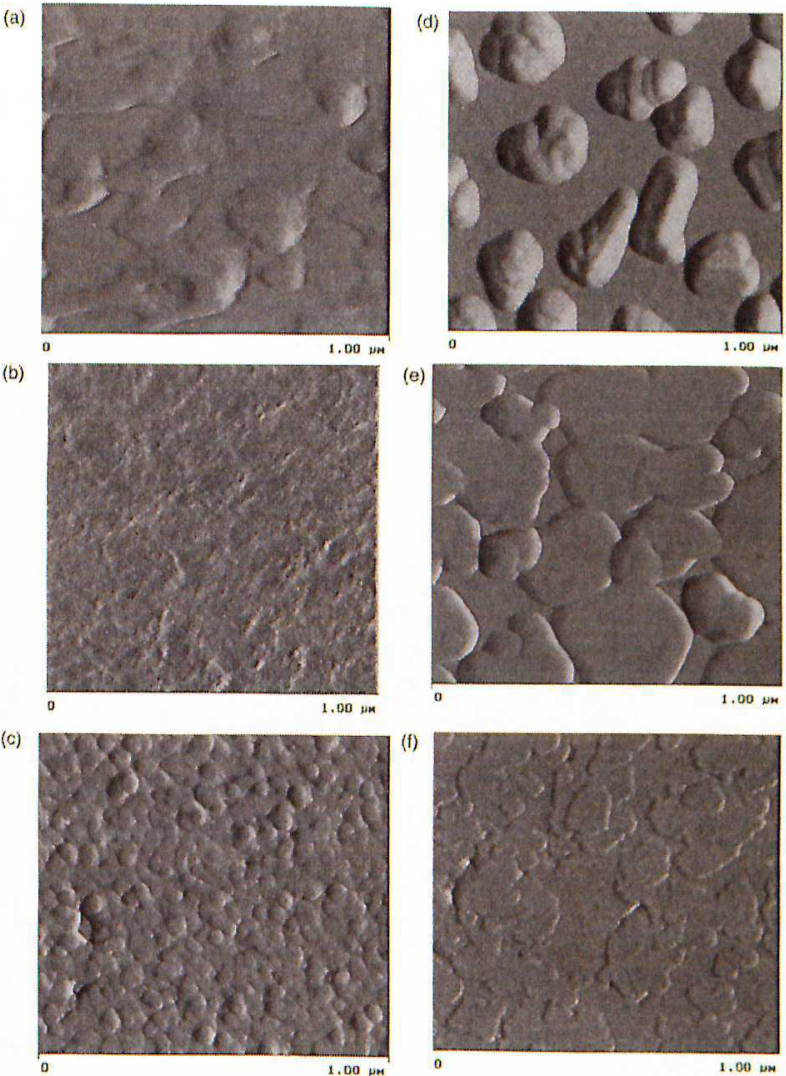

Figure 8: $C$ film growth on mica. After an average deposition of (a) two molecular layers, (b) four molecular layers, (c) seven molecular layers, at a substrate temperature of $20^{\circ} \mathrm{C}$; (d) two molecular layers, (e) four molecular layers, $(f)$ seven molecular layers, at a substrate temperature of $250^{\circ} \mathrm{C}$.

growth experiment, the $\mathrm{C}_{60}$ molecule would be expected to have a sub-eV translational energy as it approaches the surface. This slow approach means that weaker, long-range forces dominate the interaction process. As a result we have adapted these potentials by adding long-ranged pairwise interactions between the $C_{60}$ molecules themselves and the $\mathrm{C}_{60}$ molecules and the $\mathrm{Si}$ substrate. The interactions between the $\mathrm{C}_{60}$ molecules 
(a)

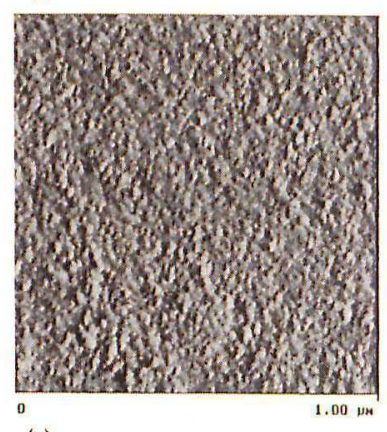

(c)

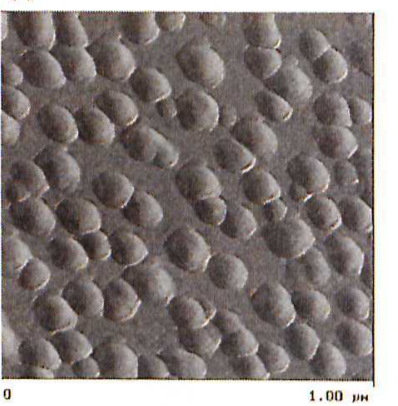

(b)

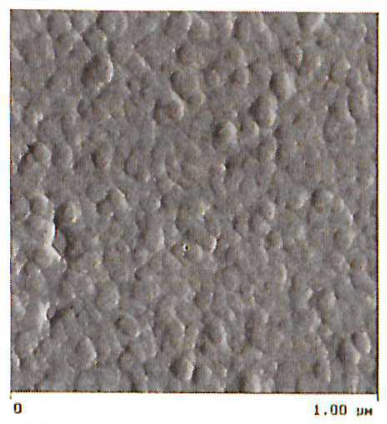

(d)

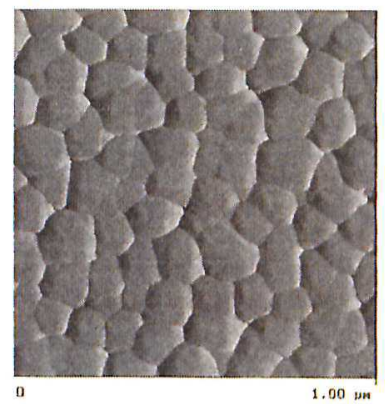

Figure 9: $C_{60}$ film growth on quartz. After an average deposition of (a) two molecular layers, (b) ten molecular layers, at a substrate temperature of $20^{\circ} \mathrm{C}$; (c) two molecular layers, (d) ten molecular layers, at a substrate temperature of $250^{\circ} \mathrm{C}$.

are modelled using a potential due to Girifalco [12]. This is a great advantage over using a long-ranged pair potential beween individual $\mathrm{C}$ atoms, as it reduces the number of long-range interactions by a factor of about 360. The simulations shown in Figure 10 were carried out using just the covalent part of the potential without the longer range Van der Waals terms.

We have also produced parameters for a potential [13] to approximate the interaction between surface silicon atoms and fullerenes during $\mathrm{C}_{60}$ film growth. The interaction beween the undercoordinated surface atoms on the Si $\{100\}(2 \times 1)$ or $\{111\}(7 \times 7)$ surfaces and the fullerene involves charge transfer from the substrate to the molecule and is therefore not modelled by the usual covalent potential. The potential correctly predicts the favoured binding site for an isolated $\mathrm{C}_{60}$ molecule to be at the centre of four dimer pairs on the $\{100\}(2 \times 1)$ surface. This is the position which would correspond to fourfold symmetry.

The simulation is started with a bare $\mathrm{Si}\{100\}(2 \times 1)$ surface. Periodic boundary conditions are applied to the sides of the surface, with free boundary conditions in the vertical direction. Two sizes of surface were used. The first surface was approximately $5.4 \mathrm{~nm} \times 5.4 \mathrm{~nm}$ and the second $4.6 \mathrm{~nm} \times 4.6 \mathrm{~nm}$. The latter size was chosen because its periodicity fits exactly a size that would allow either perfect four-fold or six-fold symmetric growth to occur. The target is 5 layers deep but the bottom two layers of the target were kept fixed. Simulations were carried out with the target held fixed at both 300 and $600 \mathrm{~K}$. Fullerenes were introduced into the simulation, with an internal energy corresponding to the sublimation temperature of $600 \mathrm{~K}$ for $\mathrm{C}_{60}$. A further $0.1 \mathrm{eV}$ kinetic energy was added to the centre of mass of each fullerene, with the velocity in a random direction. Experimentally, fullerenes are deposited at a rate of approximately 1 monolayer per minute. This is far too long a timescale to be simulated by MD. To reduce the simulation time, we introduce a new fullerene 2 ps after the last $C_{60}$ has undergone a collision that stops its vertical motion. Once fullerenes are on the surface, they are coupled to a thermal bath [8] corresponding to the target temperature. Any reflected fullerenes are removed from the simulation. Further examples of the growth of $\mathrm{C}_{60}$ films are given in reference [14].

Figure 10 contains snapshots from a simulation of growth on the surface at $20^{\circ} \mathrm{C}$. The fullerene are mobile in the first layer, especially up and down the dimer rows, and congregate into groups. The combination of the $\mathrm{Si}-\mathrm{C}_{60}$ interactions and interactions between $\mathrm{C}_{60}$ results in the first monolayer being characterised by mostly cubic but with some hexagonal stacking. This mixture of cubic and hexagonal structures is in agreement with the STM results shown in Figure 7 and
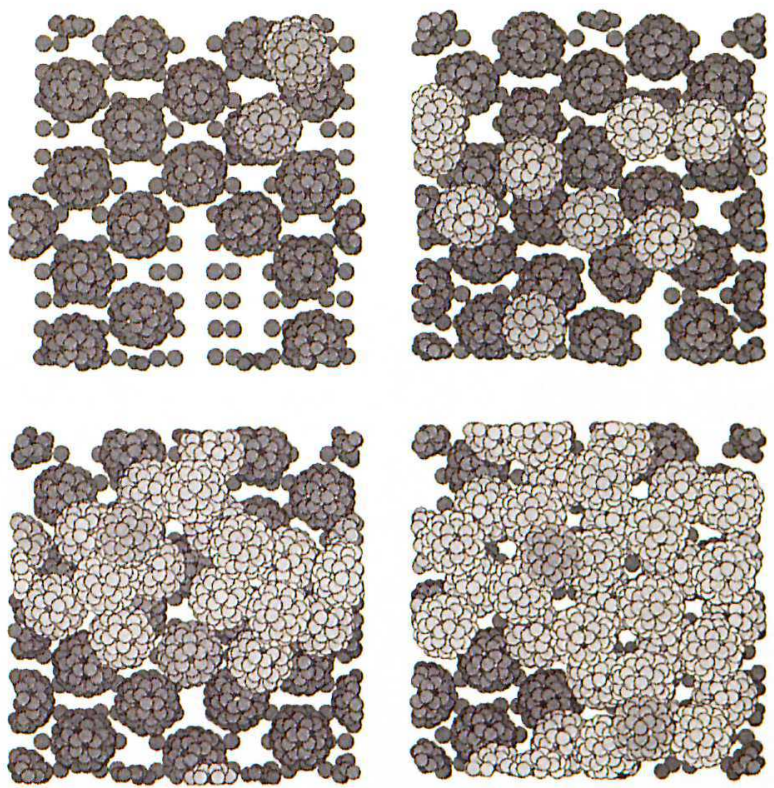

Figure: 10 (a) Computer simulation of the initial stages of growth of $C_{60}$ on Si $\{100\}(2 \times 1)$ for a lattice $4.6 \mathrm{~nm}$ at $20^{\circ} \mathrm{C}$. Note the mainly close-packed stacking.
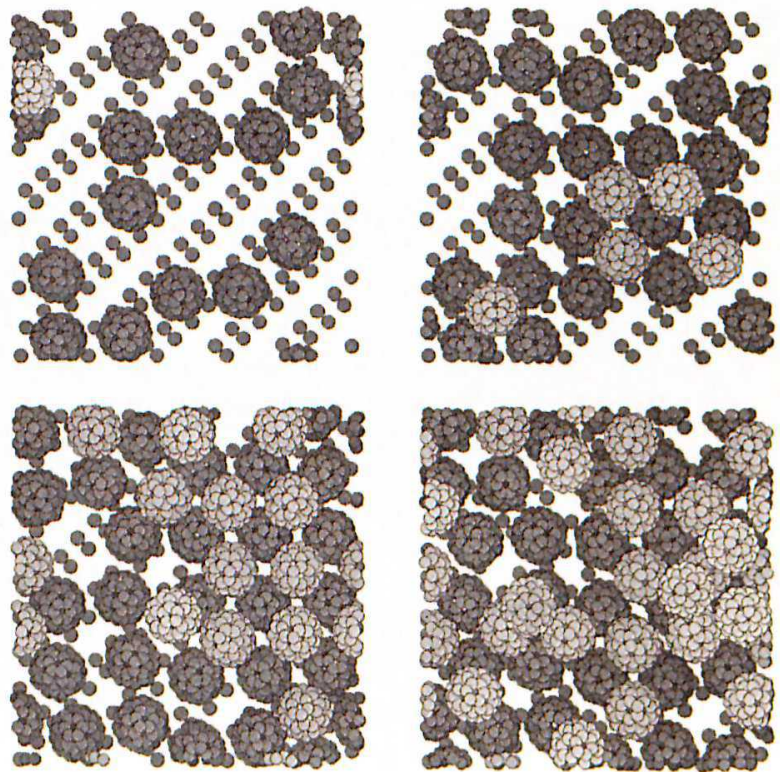

Figure 10 (b) growth on a lattice $5.4 \mathrm{~nm} \times 5.4 \mathrm{~nm}$ at $20^{\circ} \mathrm{C}$. Note the mixture of $(3 \times 4)$ and $(4 \times 4)$ stacking. 
reported in the literature $[9,15]$. As more molecules accumulate on the surface, they congregate in groups and the film becomes more stable. Molecules that impact above a closely packed region in the first monolayer can settle in hollows between molecules. The $\mathrm{C}_{60}$ 's are stable in these positions and we observe no migration of the molecules from these sites to the uncovered parts of the surface. As a result, the second layer begins to grwo before the first monolayer is complete.

\section{Conclusions}

The combination of STM/AFM together with computer simulation are powerful new tools for analysing growth at crystal surfaces. However, crystal growth is not the only example of where the combination of these two techniques can be effectively used. Other examples include irradiation damage on surfaces and indentation problems. Ab-initio molecular dynamics [16] offers even more exciting possibilities in the study of chemical reactions and catalysis.

\section{Acknowledgement}

Asta Richter was in receipt of a visiting professor's travel grant from Loughborough University which enabled this paper to be written. We gratefully acknowledge AFM micrographs of Ronald Ries and Theresa Neumann, TFH Wildau. We also acknowledge Roger Webb of Surrey University for useful disussions.

\section{References}

(1] G. Binnig, H. Rohrer, Ch. Gerber and E. Weibel, Phys. Rev. Lett. 4957 (1982).

[2] S. N. Magonov, Appl. Spectroscopy Rev. $28(1,2) 1(1993)$.

[3] F. C. Frank, Growth and Perfection of Crystals, Wiley, (1959).

14] T. Toffoli and N. Margolus, Cellular Automata Machines, MIT Press, (1987).

[5] R.Q. Hwang, J. Schröder, C. Günther and R. J. Behm, Phys. Rev. Lett. 673279 (1991).

[6] A. Richter (unpublished)

[7] T. A. Whitten and L. M. Sander, Phys. Rev. Lett. 471400 (1981).

[8] H. J. C. Berendsen, J. P. M. Postma, W. F. van Gunsteren, A. DiNola \& J. R. Haak, J.Chem. Phys. 81, 3684 (1984).

[9] X. D. Wang, T. Hashizume, H. Shinohara, Y. Saito, Y. Nishina and T. Sakurai, Phys. Rev. B. 475923 (1993).

[10] J. Tersoff, Phys. Rev. Lett. 56632 (1986); Phys. Rev. Lett. 61 2879 (1988); Phys. Rev. B. 395566 (1989).

[11] D. W. Brenner, Phys. Rev. B. 429458 (1990); Phys. Rev. B. 461948 (1992).

[12] L. A. Girifalco, J. Phys. Chem. 96858 (1992).

[13] R. Smith and K. M. Beardmore, Thin Solid Films (1996) in press

[14] K. M. Beardmore, R. Smith, A. Richter and B. Winzer, J. Mol. Modelling (1996) in press.

[15] T. Hashizume, X. D. Wang, Y. Nishina, H. Shinohara, Y. Saito, Y. Kuk and T. Sakurai, Jpn. J. Appl. Phys. 31 L880 (1992)

[16] M. J. Gillan, New Scientist 3/4/93 p35 (1993).

\section{Verfasser}

Prof. Dr. rer. nat. Asta Richter

Technische Fachhochschule Wildau

Fachbereich Physikalische Technik

D-15745 Wildau

Tel. +49 (0) 3375 / 507-219

Prof. Dr. Roger Smith

Loughborough University

Mathematical Sciences Department

Loughborough

Leicestershire, LE11 3TU, UK 